\title{
Economic growth and business cycles: a critical comment on detrending time series
}

\author{
Schenk-Hoppé, Klaus Reiner
}

\begin{abstract}
In this paper we pursue an approach based on economic theory to illustrate possible shortcomings of widely used detrending methods. We analyze a simple model of economic growth and business cycles in which investment and technical progress are stochastic. The Hodrick-Prescott and the BaxterKing filter are shown to detect spurious business cycles that are not related to actual cycles in the model. Our results cast doubts on the validity of commonly accepted stylized business cycle facts. We also discuss the relation of business cycle dating based on indicators of economic activity, as applied, for example, by the National Bureau of Economic Research, and the detrending results
\end{abstract}

DOI: https://doi.org/10.2202/1558-3708.1072

Posted at the Zurich Open Repository and Archive, University of Zurich ZORA URL: https://doi.org/10.5167/uzh-154034

Journal Article

Published Version

Originally published at:

Schenk-Hoppé, Klaus Reiner (2001). Economic growth and business cycles: a critical comment on detrending time series. Studies in nonlinear dynamics and econometrics, 5(1):Article 6.

DOI: https://doi.org/10.2202/1558-3708.1072 


\section{Studies in Nonlinear Dynamics \& Econometrics}

Volume 5 , Issue $1 \quad 2001 \quad$ Article 6

\section{Economic Growth and Business Cycles: A Critical Comment on Detrending Time Series}

Klaus R. Schenk-Hoppé

University of Zurich

ISSN: $1558-3708$

Studies in Nonlinear Dynamics \& Econometrics is produced by The Berkeley Electronic Press (bepress). All rights reserved. 


\title{
Economic Growth and Business Cycles: A Critical Comment on Detrending Time Series
}

\author{
Klaus Reiner Schenk-Hoppé \\ Institute for Empirical Research in Economics \\ University of Zurich, Switzerland \\ klaus@iew.unizh.ch
}

\begin{abstract}
In this paper we pursue an approach based on economic theory to illustrate possible shortcomings of widely used detrending methods. We analyze a simple model of economic growth and business cycles in which investment and technical progress are stochastic. The Hodrick-Prescott and the Baxter-King filter are shown to detect spurious business cycles that are not related to actual cycles in the model. Our results cast doubts on the validity of commonly accepted stylized business cycle facts. We also discuss the relation of business cycle dating based on indicators of economic activity, as applied, for example, by the National Bureau of Economic Research, and the detrending results.
\end{abstract}

Keywords. stochastic growth, detrending, spurious business cycles

Acknowledgments. I benefited from comments of the participants of the 8th Annual Meeting of the Society for Nonlinear Dynamics and Econometrics. I am grateful to Hermann Garbers, Mordecai Kurz, and Rafael Lalive D'Epinay for helpful discussions. The referees' comments helped to distinctly improve the first draft of this paper.

\section{Introduction}

Business cycles can be defined as deviations of macroeconomic data from an underlying trend that is not, however, observable in general. Since the decomposition of a time series into a trend and a remaining "cyclic" part is in principle arbitrary, any attempt to identify or approximate business cycles has to be based on economic theory, which enables one to break open the above-mentioned circularity of specifying one unobservable variable with the other.

There is a large strand of literature, in particular that due to research associated with the real business cycle school (Stadler 1994), which assumes that the trend is smooth and that all fluctuations are driven by small transient productivity shocks. Starting from this assumption, aggregate data such as output and employment are detrended using the Hodrick-Prescott filter and, more recently, the band-pass filter by Baxter and King (1999). The well-known stylized facts of the thus-specified business cycles provide a benchmark for any business cycle model (see, e.g., King and Rebelo 1999, Danthine and Donaldson 1993, and Stock and Watson 1999).

Both the assumptions imposed and the detrending methodology applied have undergone thorough inspections and recently have faced severe criticisms on two grounds. On the one hand, detrending of time series with the Hodrick-Prescott filter is shown to produce business cycle dynamics even if none are present 
in the original (artificial) time series (see, e.g., Cogley and Nason 1995, Harvey and Jaeger 1993, and Jaeger 1994, which carry out spectral analyses of structural time-series models). Using actual U.S. macroeconomic data, Canova (1998a) and Gregory and Smith (1996) find that most "stylized facts" are sensitive to the particular filter applied (see also Nelson and Kang 1981, which is seminal).

On the other hand, recent studies provide evidence that the time series of U.S. GDP is not dominated by a smooth trend. Nelson and Plosser (1982) and Murray and Nelson (2000) claim that permanent shocks dominate. Fatás (2000) questions the hypothesis of a smooth trend on the grounds of the empirical fact that long-term growth rates and persistence of output fluctuations have a strong positive correlation.

Blanchard and Fischer (1989) remarked that "macroeconomists are, and should be, schizophrenic about the use of time series methods" (6). Recent discussions, as manifested, for example, in Burnside's (1998) comment on Canova 1998a and Canova's (1998b) reply, cast doubts on the validity of the claim that the economics profession is fully aware of the potential pitfalls in using detrending methods.

This paper pursues a new direction in the study of the shortcomings of widely used detrending methods. Whereas in the above-mentioned literature either empirical macroeconomic data or structural time-series models have been employed, we propose and pursue a theory-based approach. The advantage of our approach is twofold. First, the statistical properties of the time series generated by the underlying model are completely known, whereas there is disagreement among econometricians about the actual statistical properties of macroeconomic time series. Moreover, the trend and the "cyclic" component of the time series are explicitly given by the model. Second, the structure of the decomposition of the time series into trend and cycle is not merely assumed (as in structural time-series models) but based on economic theory. Moreover, we can compare the qualitative dynamical behavior of the "cyclic" part of the original stochastic economic model with that of the detrended time series of the model and thus go beyond a mere study of the statistical properties.

The model considered here is a neoclassical growth model with stochastic technical progress and stochastic investment. It takes the form of a stochastic difference equation. The first process is the main source of long-run growth, and the latter is the main source of the short-run fluctuations. Any correlation of both sources of fluctuations can be allowed for. Using results due to Schenk-Hoppé and Schmalfuss (2001), we can completely determine the dynamical behavior and the statistical properties of the time series of this model. In particular, we can characterize the dynamics of the model, which is stochastic and nonlinear, without any approximations such as log-linearization. In particular, for each realization of the exogenous stochastic processes, the sample paths of all initial capital intensities are identical in the long run. This property ensures, for example, that the numerical simulation of the long-run dynamics of the model yields reliable results.

Assuming that technical progress is driven by a stationary process of innovations, it turns out that the trend of the stochastic capital intensity is a difference-stationary process. We study different scenarios with respect to the statistical properties of the two sources of randomness and thus can precisely quantify the erratic results of both the Hodrick-Prescott and the Baxter-King filters. Some qualitative properties of the detrending methods are also analyzed and compared with the business cycle-dating methodology based on indicators of economic activity like that applied by the National Bureau of Economic Research (NBER).

We find that even if technical progress is smooth and investment shocks are small and independent of the trend, these two detrending methods generate spurious business cycles. The higher the persistence of shocks, because of smaller rates of depreciation, the more pronounced is the misspecification of the business cycles.

The remainder of the paper is organized as follows. Section 2 presents the stochastic model of economic growth and business cycles and provides an analysis of its dynamics; in particular, existence and uniqueness of a globally stable random fixed point of the capital intensity is proved. A numerical study of the model is carried out in Section 3. There, the actual business cycles of the model and the result of detrending methods are compared. Section 4 concludes. 


\section{A Stochastic Economy}

The starting point of our study of detrending methods is a theoretical economic model of growth and business cycles with stochastic technical progress and stochastic fluctuations of investment. The model is strongly influenced by the seminal work of Solow (1956) and Swan (1956). The technology is described by a neoclassical production function, technical progress is labor-augmenting, and the investment-consumption decision of households is not explicitly modeled but assumed. We enrich this basic model by allowing for stochastic technical progress and a stochastic saving rate.

The analysis of the model applies random dynamical systems theory (cf. Arnold 1998). The main result on the long-run dynamics relies on previous work due to Schenk-Hoppé and Schmalfuss (2001) and Schenk-Hoppé (2001a). Under the assumption that the stochastic fluctuations are ergodic and that the production function satisfies an Inada-type condition, we can show that all sample paths of capital intensities asymptotically follow the same trajectory. This result enables us to derive the true decomposition of the time series of the model into a trend and a cyclic part as well as the statistical properties of the two components.

We consider an economy in which a single homogeneous good is produced at any period in time. The good can be either consumed or used as capital input. Two factors, capital and labor, are needed in the production process, described by the linear homogeneous production function

$$
Y_{t}=F\left(K_{t}, a_{t} L_{t}\right)
$$

where $K_{t} \geq 0$ is the capital stock at the beginning of period $t, a_{t} L_{t} \geq 0$ is the efficient labor supply, that is, $a_{t}$ is a measure of technical progress at time $t$, and $L_{t}$ is aggregate labor supply. Technical progress is labor-augmenting. We assume that $(K, L) \mapsto F\left(K, a_{t} L\right)$ is neoclassical, exhibits constant returns to scale, and satisfies the Inada conditions for each possible realization of the exogenous variable $a_{t}$ (cf. Barro and Sala-i-Martin 1995, sec. 1.2.1). Households do not have disutility from work and inelastically supply their total endowment of labor. We further assume a closed economy, that is, the endowment of capital at the beginning of period $t+1$ is equal to the resources not consumed in the preceding period. Thus the law of motion of the capital stock is given by

$$
K_{t+1}=F\left(K_{t}, a_{t} L_{t}\right)+\left(1-\delta_{t}\right) K_{t}-C_{t}
$$

where $C_{t}$ denotes aggregate consumption in period $t$ and $\delta_{t}$ is the rate of depreciation.

Analogously to the standard Solow-Swan model we assume that each household consumes a fraction $1-s_{t}$ of the total output in every period in time, that is, $C_{t}=\left(1-s_{t}\right) F\left(K_{t}, a_{t} L_{t}\right)$.

We make the following specific assumption on the process of technical innovations.

Assumption 2.1. The evolution of the efficient labor supply, $a_{t} L_{t}$, is given by $a_{t+1} L_{t+1}=\left(1+n_{t}\right) a_{t} L_{t}$, and the exogenous variable $\left(n_{t}, \delta_{t}, s_{t}\right)$ is an ergodic process.

Appropriate assumptions on the range of values for these processes are imposed below. For the moment it is sufficient to assume $a_{t} L_{t}>0$ for all $t$.

Define the capital per efficient unit of labor $k_{t}=K_{t} /\left(a_{t} L_{t}\right)$, henceforth called capital intensity. Under assumption 2.1, Equation (2.1) yields the following stochastic law for the capital intensity:

$$
k_{t+1}=\frac{K_{t+1}}{a_{t+1} L_{t+1}}=\frac{\left(1-\delta_{t}\right) K_{t}+s_{t} F\left(K_{t}, a_{t} L_{t}\right)}{\left(1+n_{t}\right) a_{t} L_{t}}=\frac{\left(1-\delta_{t}\right) k_{t}+s_{t} f\left(k_{t}\right)}{1+n_{t}}
$$

where $f(k):=F(k, 1)$ is the intensity form of $F$ (also a neoclassical production function).

We model the ergodic process $\left(n_{t}, \delta_{t}, s_{t}\right)$ by an ergodic dynamical system $(\Omega, \mathcal{F}, \mathbb{P}, \theta)$. That is, the probability space $(\Omega, \mathcal{F}, \mathbb{P})$ is the sample path space, and $\theta$ is the shift map. In this notation, the stochastic 
law becomes

$$
k_{t+1}=h\left(\theta^{t} \omega, k\right):=\frac{\left(1-\delta\left(\theta^{t} \omega\right)\right) k_{t}+s\left(\theta^{t} \omega\right) f\left(k_{t}\right)}{1+n\left(\theta^{t} \omega\right)}
$$

For a given initial state $k_{0}$ of the capital intensity and any sample path of the exogenous stochastic process, Equation (2.2), the random difference equation, defines a sample path of the capital intensity. Equation (2.2) is henceforth called the stochastic Solow model.

Equation (2.2) generates a random dynamical system on the state space $\mathbb{R}_{+}$in the following sense. Define

$$
\varphi(t, \omega, k)= \begin{cases}h\left(\theta^{t-1} \omega\right) \circ \cdots \circ b(\omega) k & \text { for } t \geq 1 \\ k & \text { for } t=0 \\ h\left(\theta^{t} \omega\right)^{-1} \circ \cdots \circ b\left(\theta^{-1} \omega\right)^{-1} k & \text { for } t \leq-1\end{cases}
$$

where $h(\omega):=h(\omega, \cdot): \mathbb{R}_{+} \rightarrow \mathbb{R}_{+} . \varphi(t, \omega, k)$ is the state of the stochastic system (2.2) at time $t$ that has been started at $k_{0}=k$ under the perturbation determined by $\omega$.

The family of maps $\varphi(t, \omega, k)$ is called a random dynamical system. That is, $\varphi: \mathbb{Z} \times \Omega \times \mathbb{R}_{+} \rightarrow \mathbb{R}_{+}$, $(t, \omega, k) \mapsto \varphi(t, \omega, k)$ is a measurable mapping such that $\varphi(0, \omega)=\mathrm{id}_{\mathbb{R}_{+}}$and $\varphi(s+t, \omega)=\varphi\left(t, \theta^{s} \omega\right) \circ \varphi(s, \omega)$ for all $s, t \in \mathbb{Z}$ and all $\omega \in \Omega$. Note that these properties replace the flow property of a deterministic dynamical system that is generated by the iteration of a map. Obviously, $\varphi(t, \omega)$ inherits the regularities (such as continuity or smoothness) of $b$ for $t \geq 0$ and of $b^{-1}$ for $t \leq 0$.

We define the concept of a fixed point, which is central to our subsequent analysis of the model.

Definition 2.1. A random fixed point of the random dynamical system $\varphi$ generated by the stochastic Solow model is a random variable $k^{\star}: \Omega \rightarrow \mathbb{R}_{+}$such that almost surely

$$
k^{\star}(\theta \omega)=\varphi\left(1, \omega, k^{\star}(\omega)\right):=h\left(\omega, k^{\star}(\omega)\right)
$$

We are now in a position to state the main auxiliary result of this section. A proof can be found in Schenk-Hoppé and Schmalfuss 2001.

Theorem 2.1. Assume that $\left.\delta(\omega) \in\left[\delta_{\min }, \delta_{\max }\right] \subset[0,1], n(\omega) \in\left[n_{\min }, n_{\max }\right] \subset\right]-1, \infty[$, and $\left.\left.s(\omega) \in\left[s_{\min }, 1\right] \subset\right] 0,1\right]$. Assume further that $f$ is non-negative, increasing, strictly concave, and continuously differentiable.

Suppose that

(i) $\delta_{\max }+n_{\max }>0$

(ii) $0 \leq \lim _{k \rightarrow \infty} f^{\prime}(k)<\frac{\delta_{\max }+n_{\max }}{s_{\min }}<\lim _{k \rightarrow 0} f^{\prime}(k) \leq \infty$

(iii) $\mathbb{E} \log \frac{1-\delta(\omega)+s(\omega) f^{\prime}(\bar{k})}{1+n(\omega)}<0$

where $\bar{k}:=\bar{k}\left(\delta_{\max }, n_{\max }, s_{\min }\right)$ is the positive fixed point of the deterministic Solow model with respective parameters, that is, (2.2) with $\delta(\omega) \equiv \delta_{\max }, n(\omega) \equiv n_{\max }$, and $s(\omega) \equiv s_{\min } . \bar{k}$ is well-defined and unique by the assumptions on $f$ and conditions ( $i$ ) and (ii).

Then there exists a unique positive random fixed point $k^{\star}$ for the stochastic Solow model (2.2). $k^{\star}$ is asymptotically stable, measurable with respect to the past, and globally attracting on $\mathbb{R}_{++}$, that is, for all $k>0$, $\left|\varphi(t, \omega, k)-k^{\star}\left(\theta^{t} \omega\right)\right| \rightarrow 0$ as $t \rightarrow \infty$ a.s.

The result ensures that the long-run behavior of all sample paths is uniquely determined by the random fixed point $k^{\star}$. For each initial capital intensity, the sample path asymptotically moves jointly with $t \mapsto k^{\star}\left(\theta^{t} \omega\right)$. The dynamics is thus governed by the ergodic process $k^{\star}\left(\theta^{t} \omega\right)$. 
Recall that the evolution of the aggregate capital stock is described by the (nonstationary) function $K_{t}=a_{t} L_{t} k_{t}=\prod_{u=0}^{t-1}\left(1+n\left(\theta^{u} \omega\right)\right) a_{0} L_{0} k_{t}$ for each initial value of the efficient labor supply $a_{0} L_{0}$. The first part is difference-stationary after taking logarithms because $\log \left(a_{t+1} L_{t+1}\right)=\log \left(1+n\left(\theta^{t} \omega\right)\right)+\log \left(a_{t} L_{t}\right)$, by definition, and $n\left(\theta^{t} \omega\right)$ is ergodic and thus stationary.

We therefore obtain the following result.

Corollary 2.1. Fix any initial state of efficient labor supply $a_{0} L_{0}>0$. Then the sample path of the capital stock $K_{t}=a_{t} L_{t} k_{t}$ is governed asymptotically by the sample path $t \mapsto a_{t} L_{t} k^{\star}\left(\theta^{t} \omega\right)$ for each initial state $K_{0}>0$ and for almost all $\omega \in \Omega$.

The asymptotic motion of the logarithm of the capital stock can therefore be decomposed into a difference stationary part and an ergodic part with expected value zero:

$$
\log K_{t}=\left[\log \left(a_{t} L_{t}\right)+\mathbb{E} \log k^{\star}\right]+\left[\log k^{\star}\left(\theta^{t} \omega\right)-\mathbb{E} \log k^{\star}\right]
$$

The two bracketed terms in Equation (2.5) are referred to as trend and cycle, respectively.

For each realization of the exogenous process, a variation of the initial state of efficient labor supply $a_{0} L_{0}$ results in a parallel translation of the sample path of the capital stock. We therefore can and do assume $a_{0} L_{0}=1$. The decomposition (2.5) can be written as

$$
\log K_{t}=\left[\sum_{u=0}^{t-1} \log \left(1+n\left(\theta^{u} \omega\right)\right)+\mathbb{E} \log k^{\star}\right]+\left[\log k^{\star}\left(\theta^{t} \omega\right)-\mathbb{E} \log k^{\star}\right]
$$

We discuss the properties of the two stochastic processes defined in the decomposition (2.5)—trend and cycle-in turn. The trend is a nonstationary yet difference-stationary process. Its systematic contribution to the growth of the capital stock is given by $\mathbb{E} \log K_{t}=t \mathbb{E} \log (1+n)+\mathbb{E} \log k^{\star}$. The fluctuations of the trend are completely attributable to stochastic variations of the efficient labor supply, that is, to $\log \left(a_{t} L_{t}\right)$.

The cycle is an ergodic process with mean zero. No systematic tendency of the growth of $\log K_{t}$ is caused by this part of the decomposition. The fluctuations of the cycle are stationary and stem from the variation of the capital intensity $k^{\star}$. The cyclic part therefore depends on the process describing the stochastic investment as well as on the process of innovation. The latter causes an indirect dependence of the cycle on the state of technical progress.

Put differently, the ergodic investment process causes fluctuations only in the cycle part, whereas the ergodic innovation process triggers fluctuations of both trend and cycle.

The decomposition of the capital stock process defined in Corollary 2.1 also yields a decomposition of the total output. We have

$$
\log Y_{t}=\left[\log \left(a_{t} L_{t}\right)+\mathbb{E} \log f\left(k^{\star}\right)\right]+\left[\log f\left(k^{\star}\left(\theta^{t} \omega\right)\right)-\mathbb{E} \log f\left(k^{\star}\right)\right]
$$

It is left to the reader as an easy exercise to derive the decomposition of the investment, the interest and wage rate (which are both ergodic), and the capital and labor share (which have decompositions similar to that given above). We will not need these processes in further study.

In summary, we have set up and completely analyzed the model in this section. The main result is the decomposition of the motion of the capital stock into trend and cycle. Both components are derived by the principles of economic theory and have clear-cut economic interpretations. The statistical properties of both components have also been described in detail.

\section{Numerical Analysis}

In this section we apply the Hodrick-Prescott and Baxter-King filters to time series generated by our stochastic economic model. These widely used detrending methods yield a decomposition of the stochastic aggregates into a trend and a cyclic part. We examine the relation between this decomposition and the trend and the 
cycle defined in the previous section using economic theory. In other words, we study numerically whether the Hodrick-Prescott or the Baxter-King filter detects the actual business cycles in the time series generated by the model. The main emphasis in this study is on qualitative rather than statistical properties of the cycle.

The software used in the simulations is a collection of MATLAB scripts. It is available on the web (Schenk-Hoppé 2001b). Using the software the reader can reproduce and check our results as well as analyze other interesting cases that lack of space does not permit us to present here.

Before presenting our numerical study, some remarks on the different usage of the terms "trend" and "cycle" in econometrics and economic theory are in order. In econometrics, these two notions refer to different spectral properties; that is, they are distinguished with respect to frequencies. The cycle is associated with frequencies between 4 and 32 quarters, whereas the trend is related to the lower frequencies in a time series.

In economic theory the trend refers to that part of a time series that is related to technical progress, whereas the cycle corresponds to the business cycle. The trend is commonly believed to increase steadily and to show only sluggish variations over time, whereas the business cycle is associated with economic policies that have a short- or medium-run effect on the economy. This point of view is manifested in the fact that the two components are studied in different fields, growth theory and business cycle theory, respectively. Only recently have stronger ties between these two fields started to develop through empirical and theoretical progress. However, the above-mentioned distinction, or decomposition, does not rule out any possible dependencies between both factors and their impact on the growth of an economy. Even in our simple model the innovation process exhibits an effect on the short-run fluctuations.

We need to make specific assumptions on the stochastic processes governing the evolution of innovation and investment as well as on the fundamentals.

\section{Assumption 3.1.}

(i) Labor supply is fixed and normalized to one; that is, $L_{t} \equiv 1$. The rate of depreciation $\delta$ is constant.

(ii) The process of innovation $n(\omega)$ is defined as follows: $n(\omega)=0.0075+\eta(\omega)$, where $\eta$ is the ergodic process generated by $\eta_{t+1}=A \eta_{t}+\phi_{t}$, with i.i.d. process $\phi_{t}$ being uniformly distributed on $[-\bar{\phi}, \bar{\phi}]$.

(iii) The process of investment $s(\omega)$ is defined as follows: $s(\omega)=0.25 *(1+z(\omega))$, where $z$ is the ergodic process generated by $z_{t+1}=B z_{t}+\rho_{t}$, with i.i.d. process $\rho_{t}$ being uniformly distributed on $[-\bar{\rho}, \bar{\rho}]$.

(iv) The technology is described by the Cobb-Douglas production function

$$
f(k)=k^{\alpha}, \quad 0<\alpha<1
$$

We further assume that $\phi_{t}$ and $\rho_{t}$ are independent.

The expected value of the saving rate is 0.25 , and the expected value of technical progress is about $3 \%$ per year, where we interpret each period in time as one quarter of the year in the simulation.

We study three cases. The parameter settings are chosen as follows. We fix $A=0.95, B=0.95$, $\bar{\rho}=5 * 10^{-4}$, and $\bar{\phi}=5 * 10^{-3}$ throughout the analysis. The other parameters are set as follows:

Case 1: $\alpha=0.75, \delta=0.9$.

Case 2: $\alpha=0.25, \delta=0.9$.

Case 3: $\alpha=0.25, \delta=0.1$.

We first need to ensure that Theorem 2.1 applies for the above parameter settings.

First note that $\eta(\omega) \in[-\bar{\phi} /(1-A),+\bar{\phi} /(1-A)]$ and $z(\omega) \in[-\bar{\rho} /(1-B),+\bar{\rho} /(1-B)]$ for all $\omega$. Second, elementary calculations yield the steady state of the associated deterministic model:

$$
\bar{k}\left(\delta_{\max }, n_{\max }, s_{\min }\right)=\left(s_{\min } /\left(\delta_{\max }+n_{\max }\right)\right)^{1 /(1-\alpha)}
$$


Using this expression, the contraction condition (iii) of Theorem 2.1 is satisfied if and only if

$$
\mathbb{E} \log \left(1-\delta+\alpha s(\omega) \frac{\delta+n_{\max }}{s_{\min }}\right)<\mathbb{E} \log (1+n(\omega))
$$

since $\delta$ is deterministic. The validity of Equation (3.2) in the cases 1-3 can be checked numerically (see Schenk-Hoppé 2001b).

\subsection{Case 1}

We start with a case in which production is relatively capital intensive, $\alpha=0.75$, and the rate of depreciation is high, $\delta=0.9$. The setting of the production parameter is roughly in line with empirical studies employing the deterministic Solow model.

The simulation of the stochastic economy-described in Section 2-is carried out as follows. First, the initial states of all processes are set to their expected value. Second, the model is iterated 600 periods to ensure that the sample path of the capital intensity is close to the path of the random fixed point $t \mapsto k^{\star}\left(\theta^{t} \omega\right)$. Numerical studies show that this is indeed the case for the parameter settings considered here. Third, the model is simulated for 200 periods, where a period is understood as representing a quarter of a year. Thus the data generated in the last 200 periods represent the time series of output and capital of our model economy over a time horizon of 50 years. Fourth, we calculate the actual trend and cycle for the time series of logarithms of aggregate output and capital stock, $\log \left(Y_{t}\right)$ and $\log \left(K_{t}\right)$, according to the definition given in Equations (2.5) and (2.6). Finally, we apply the Hodrick-Prescott filter HP(1600) with parameter $w=1600$ and the band-pass filter BK(6,32,12), $K=12$, introduced by Baxter and King (1999), to the time-series $\log \left(Y_{t}\right)$ and $\log \left(K_{t}\right)$.

Figures 1 and 2 depict the results for case 1. We first note that the Hodrick-Prescott and Baxter-King filters show a close correspondence. This can be observed in all simulations and is in agreement with the findings of Baxter and King (1999). We will therefore mention only the Hodrick-Prescott filter in subsequent discussions. The BK(6,32,12) filter produces a smoother line because it is a band-pass filter and also removes the higher frequency components from a time series, which is not true of the HP(1600) filter.

In Figure 1 the actual cycle of the logarithm of the total output exhibits values of roughly between $\pm 4 \%$. It is straightforward to check that this implies a maximal deviation of total output $Y_{t}$ from the true trend of about 9.5\%. One observes a clear pattern of recurrent periods in which the logarithm of the total output is above (below) its trend; the cycle is positive (negative). The length of these periods as well as the magnitude of the cycle vary over time. The longer a period, however, the larger the deviation from the trend. It is also noteworthy that the actual cycle is quite smooth. In summary, we can clearly distinguish the different features of the actual business cycle in total output in our stochastic model.

The Hodrick-Prescott filter predicts a business cycle that exhibits about the same number of booms and recessions as existent in the actual cycle. The average magnitude of the HP cycle is smaller, however, than that of the actual cycle. The dating of the cycles using the Hodrick-Prescott filter is mainly erratic. Between year 10 and 30 the HP cycle shows a behavior similar to that of the actual cycle but predates the booms and recessions by $2-3$ years.

Figure 2 depicts the results for the logarithm of the capital stock. The features of the actual cycle and the HP cycle are very similar to those discussed above, because the rate of depreciation is high. The variations caused by the innovation and investment process affect output and capital almost in the same magnitude.

\subsection{Case 2}

We next consider a case in which production is relatively intensive in human capital, $\alpha=0.25$. We keep the same rate of depreciation as in case 1 . Thus only the production parameter is changed compared to the previous case. The simulations have been carried out exactly as explained above. 


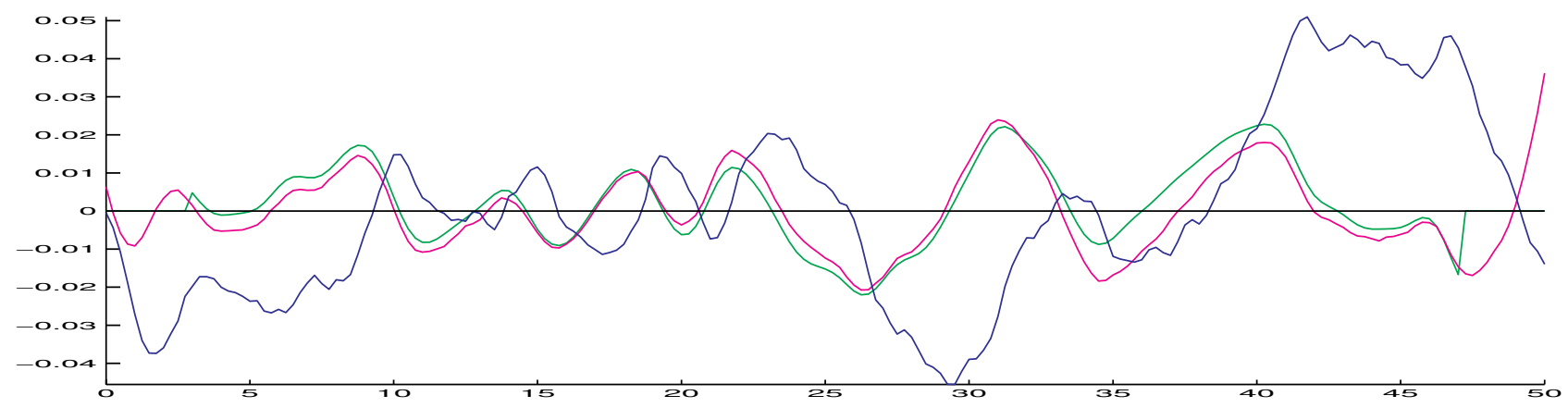

Figure 1

[Case 1] Business cycles of GDP (deviation of $\log \left(Y_{t}\right)$ from different trends): HP(1600) (magenta), BK(6,32,12) (green), actual cycle (blue).

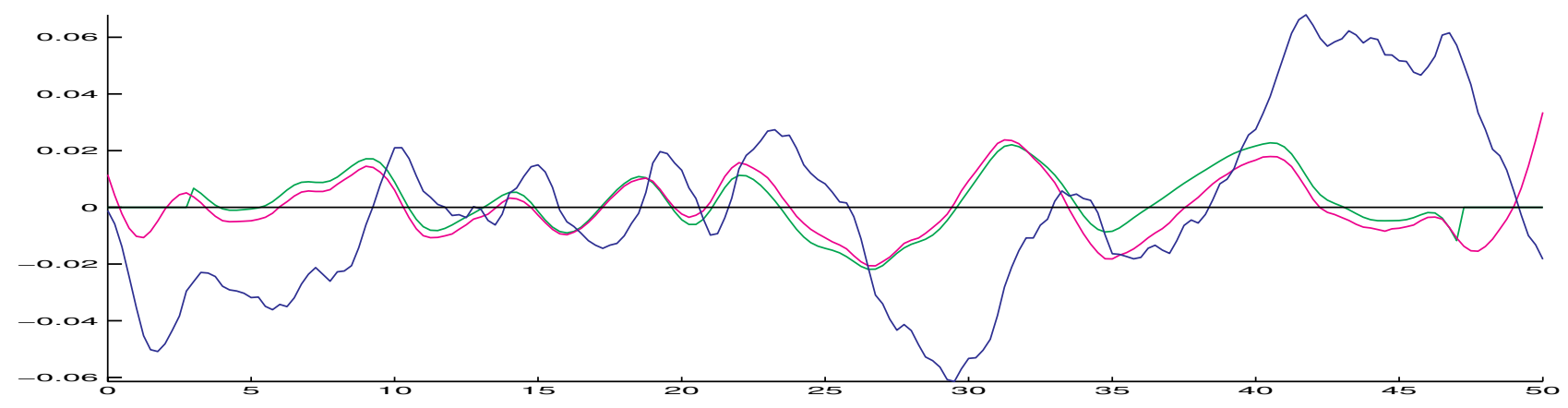

Figure 2

[Case 1] Business cycles of capital stock (deviation of $\log \left(K_{t}\right)$ from different trends): HP(1600) (magenta), BK(6,32,12) (green), actual cycle (blue).

Figures 3 and 4 depict the results for case 2 .

Because physical capital is used less intensively in the production process than human capital, the logarithm of the total output of the stochastic economy exhibits smaller deviations from the trend than in case 1. Aggregate output deviates from the actual trend by less than 1\%. There are no pronounced booms and recessions in the stochastic economy. The Hodrick-Prescott filter, however, predicts a business cycle of comparatively large magnitude. There is no relation between the HP and the actual cycle. The spurious business cycles as dated by the HP filter last roughly about 4 years.

The situation is somewhat different in the time series of the capital stock. There the actual and HP cycle are of the same magnitude. The HP cycle exhibits a correlation with the actual cycle to a certain degree. The business cycle dating is not completely erratic, though the agreement between the HP and the actual data is quite bad. Again we observe a predating of the cycle by 1 to 2 years.

\subsection{Case 3}

We now study our stochastic economy with a comparatively low rate of depreciation, $\delta=0.1$. All other parameters are set as in case 2; in particular, production is relatively intensive in human capital, $\alpha=0.25$. Again the simulations follow the same procedure as discussed above.

Figures 5 and 6 depict the results for case 3 .

Because of the assumption that capital depreciates slower than in the previous cases, the time series of the logarithm of the capital stock (Figure 6) exhibits large deviations from its trend and is relatively smooth. The 


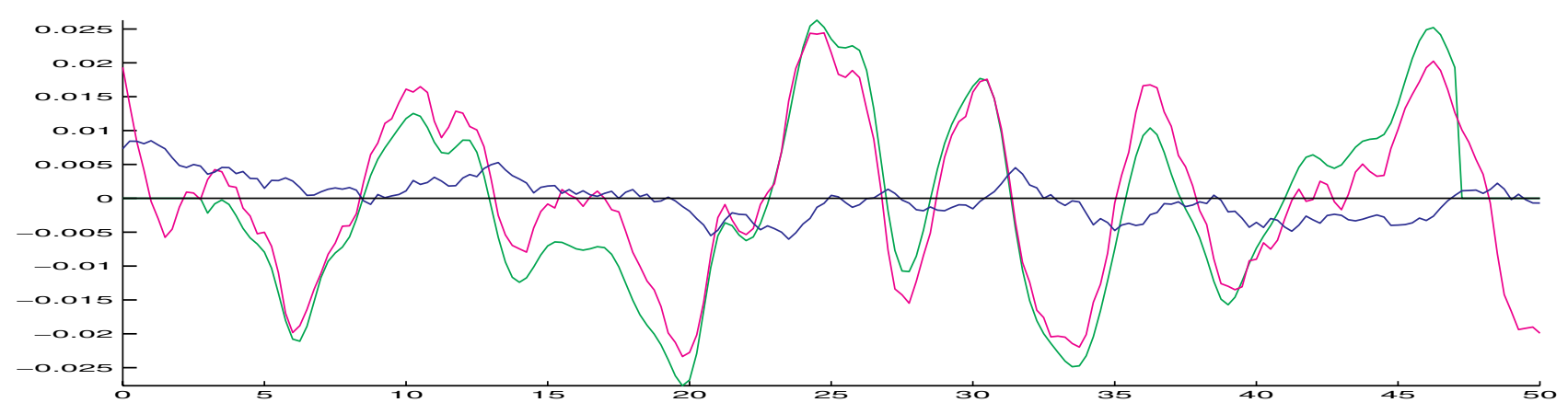

Figure 3

[Case 2] Business cycles of GDP (deviation of $\log \left(Y_{t}\right)$ from different trends): HP(1600) (magenta), BK(6,32,12) (green), actual cycle (blue).

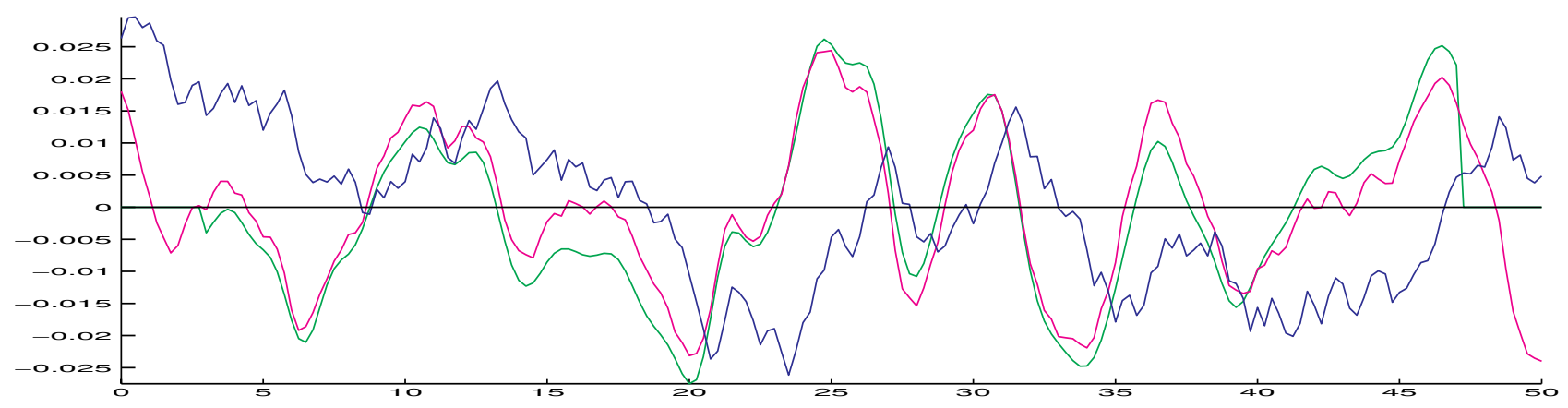

Figure 4

[Case 2] Business cycles of capital stock (deviation of $\log \left(K_{t}\right)$ from different trends): HP(1600) (magenta), BK(6,32,12) (green), actual cycle (blue).

same pattern can be observed for the actual cycle of the aggregate output (Figure 5). Since production is relatively intensive in human capital, the deviations from the trend are smaller than for the capital stock.

In Figure 5 the Hodrick-Prescott filter predicts business cycles of approximately the true magnitude for total output. The dating as well as the length of the HP business cycle is erratic. The spurious business cycles have a length of about 3 years. There is almost no relation between the HP and the actual cycle.

The result of the Hodrick-Prescott filter is even worse for the time series of the capital stock (see Figure 6). The magnitude of the HP cycle is about eight times too small. The dating of the cycle is also unrelated to the actual behavior.

\subsection{Summary of the numerical results}

We have analyzed numerically three different cases of our artificial economy. In neither case has the Hodrick-Prescott or the Baxter-King filter tracked down the actual cycle. In fact the approximation of the true cycle is very poor. In all cases both filters produced spurious cycles of an average length of about 3 to 5 years. In most cases the magnitude of the deviation of the time series from the true trend is either over- or underestimated. In case 2 (case 3) - in which production is labor intensive-the fluctuation of total output (capital stock) is predicted to be 5 times larger (smaller) than it actually is for high (low) depreciation. Any business cycle dating based on these filters leads to incorrect statements.

Of course it is mandatory to realize the causes of the observed shortcomings when applying these two widely used filters. The qualitative results in Section 2 ensured that the time series under study are integrated of order one (i.e., difference stationary). Together with the econometric results on the application of the 


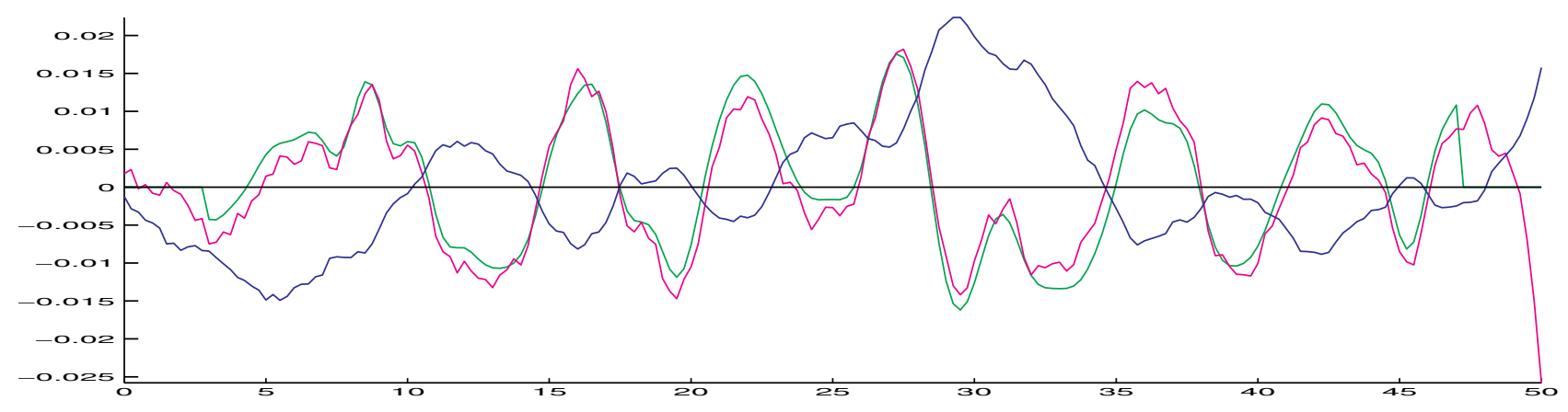

Figure 5

[Case 3] Business cycles of GDP (deviation of $\log \left(Y_{t}\right)$ from different trends): HP(1600) (magenta), BK(6,32,12) (green), actual cycle (blue).

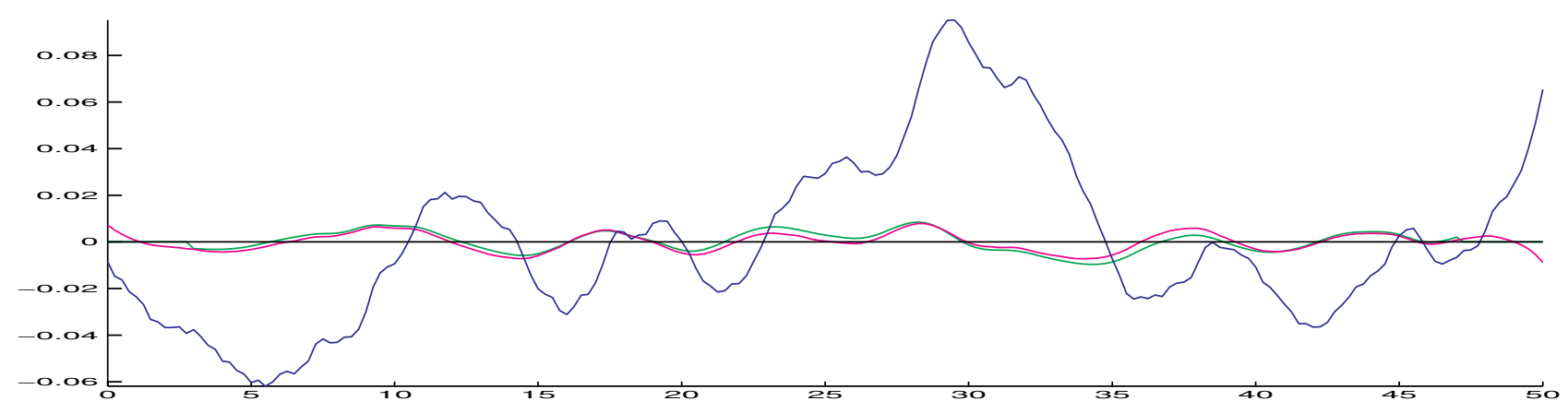

Figure 6

[Case 3] Business cycles of capital stock (deviation of $\log \left(K_{t}\right)$ from different trends): HP(1600) (magenta), BK(6,32,12) (green), actual cycle (blue).

Hodrick-Prescott filter to integrated time series-to which we have already pointed the reader in the introduction-we have a good understanding of those causes.

\subsection{NBER business cycle dating revisited}

In this section we focus on those causes of the detrending problems that can be detected by making use of the additional knowledge we have about the innovation and investment processes in our model. It will be shown that there is a strong link between the predictions of the Hodrick-Prescott (and Baxter-King) filter and the changes in economic activity in our model. Since NBER business cycle dating is based on indicators of economic activity, this observation highlights a possible explanation as to why the HP and NBER cycles exhibit a very close correspondence in empirical studies.

Figure 7 depicts the time series of the innovation process. Comparison of this figure with the time series of total output and capital stock in case 3 (Figures 5 and 6) yields the following observation. The sample path of either time series is above (below) the actual trend if the sample path of the innovation process is below (above) its expected value. This behavior is due to the definition of the trend in our model: the trend is that part of the time series that is due to technical progress. If technical progress is slowing down, then the slope of the trend decreases. If investment does not fall accordingly, the capital per efficient unit of labor increases faster than the trend. Therefore the cycle is positive during these periods, and we thus observe a boom. Clearly this definition of trend and cycle is not related to economic activity in a strict sense.

Interpreting economic activity in our model as (major) changes in the investment process, we can state the following observation. The Hodrick-Prescott (and Baxter-King) filter gives a clear-cut prediction on the 


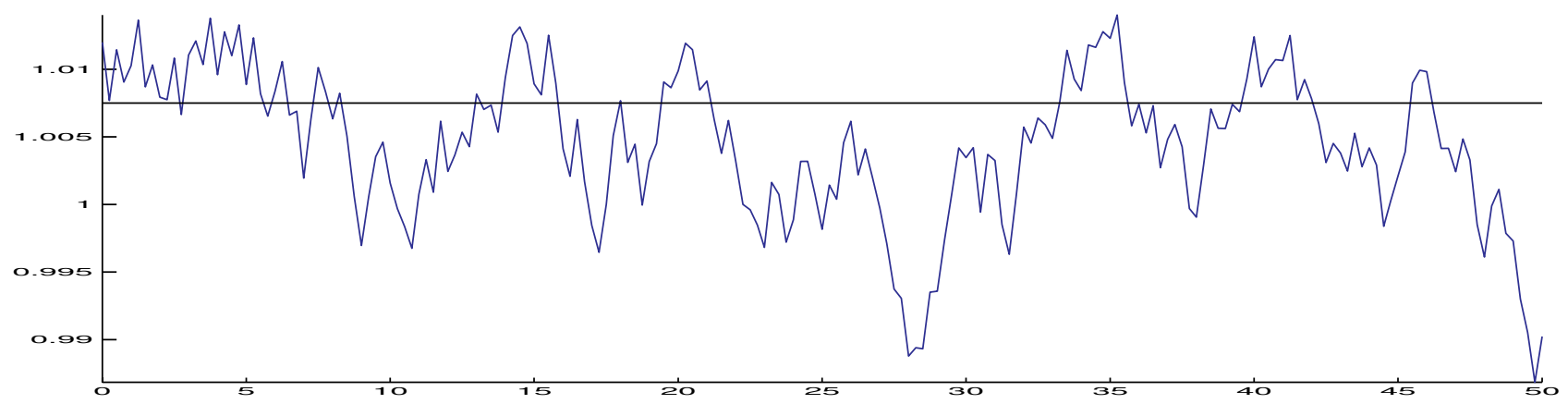

Figure 7

[Case 3] Time series of technical progress $1+n\left(\theta^{t} \omega\right)$.

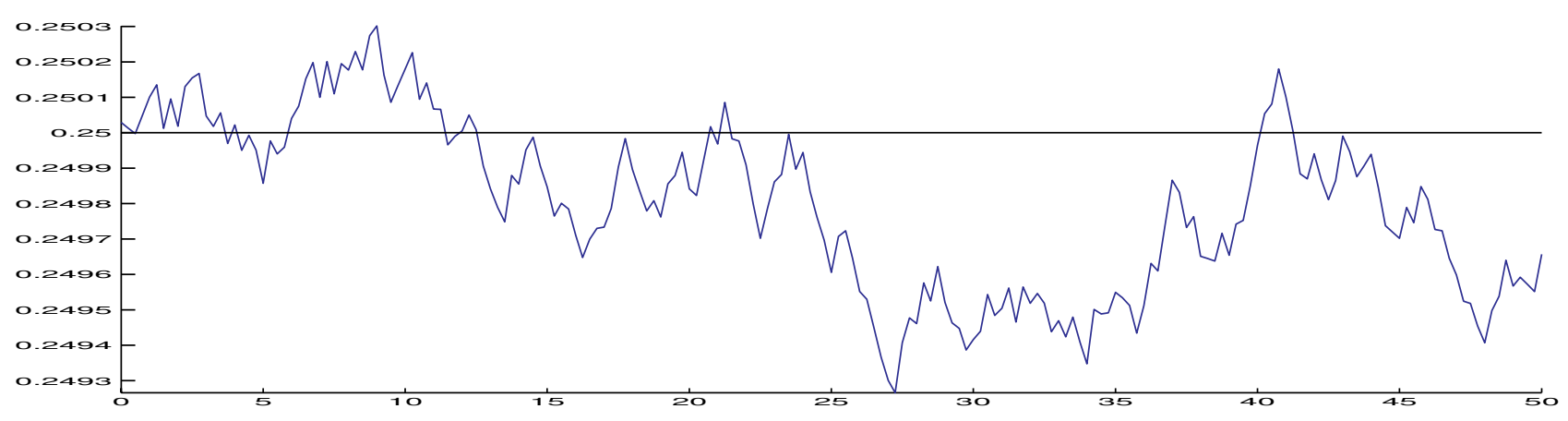

Figure 8

[Case 3] Time series of investment $s\left(\theta^{t} \omega\right)$.

changes in investment (cf. Figures 5 and 7). The HP cycle is positive throughout years 5 to 10. In this period innovation is slowing down, whereas investment is above its expected value and experiences a temporary high in year 8 . The actual cycle is negative. Similar patterns can be observed also in the period from years 28 to 35. Both processes are below their expected value throughout this period. In year 30 investment has a local minimum, whereas innovation experiences a local maximum. The HP cycle is negative but has a local minimum at year 30 . The actual cycle is positive throughout this period. Summarizing, we may state that the HP cycle is closely related to changes in the investment process.

\section{Conclusions}

This paper illustrates the dangers of detrending nonstationary macroeconomic time series by filters. It provides a critical assessment of a common practice in empirical research in which econometric methods are employed without a sound theoretical foundation. To this end we presented a model of stochastic economic growth in which the actual business cycles are not detected by the most commonly applied filters in real business cycle theory. Both the Hodrick-Prescott and the Baxter-King filters generate spurious business cycles when applied to the data generated by the model.

Our reasoning is based on economic theory, defining the trend as that part of a time series that is caused by technical progress. Our criticisms give further support to the purely econometric approaches due to Canova (1998a, 1998b), Cogley and Nason (1995), Harvey and Jaeger (1993), Jaeger (1994), and Nelson and Kang (1981), among others. We suggest that the findings of this paper and related work be understood as a motivation to strive for a new methodology (or the resuscitation of classical approaches) for determining the trend in economic growth paths. 


\section{References}

Arnold, L. (1998). Random Dynamical Systems. New York: Springer-Verlag.

Barro, R. J., and X. Sala-i-Martin. (1995). Economic Growth. New York: McGraw-Hill.

Baxter, M., and R. G. King. (1999). "Measuring business cycles: Approximate band-pass filters for economic time series." Review of Economics and Statistics, 81: 575-593.

Blanchard, O. J., and S. Fischer. (1989). Lectures on Macroeconomics. Cambridge: MIT Press.

Burnside, C. (1998). "Detrending and business cycle facts: A comment." Journal of Monetary Economics, 41: 513-532.

Canova, F. (1998a). "Detrending and business cycle facts.” Journal of Monetary Economics, 41: 475-512.

Canova, F. (1998b). "Detrending and business cycle facts: A user's guide." Journal of Monetary Economics, 41: 533-540.

Cogley, T., and J. M. Nason. (1995). "Effects of the Hodrick-Prescott filter on trend and difference stationary time series: Implications for business cycle research.” Journal of Economic Dynamics and Control, 19: 253-278.

Danthine, J. P., and J. B. Donaldson. (1993). "Methodological and empirical issues in real business cycles theory." European Economic Review, 37: 1-35.

Fatás, A. (2000). "Endogenous growth and stochastic trends." Journal of Monetary Economics, 45: 107-128.

Gregory, A. W., and G. W. Smith. (1996). "Measuring business cycles with business-cycle models." Journal of Economic Dynamics and Control, 20: 1007-1025.

Harvey, A. C., and A. Jaeger. (1993). "Detrending, stylized facts and the business cycle." Journal of Applied Econometrics, 8: $231-247$.

Jaeger, A. (1994). "Mechanical detrending by Hodrick-Prescott filtering: A note." Empirical Economics, 19: 493-500.

King, R. G., and S. T. Rebelo. (1999). "Resuscitating real business cycles.” In J. B. Taylor and M. Woodford (eds.), Handbook of Macroeconomics, vol. 1B, chapter 14. Amsterdam: Elsevier, pp. 927-1007.

Murray, C. J., and C. R. Nelson. (2000). "The uncertain trend in U.S. GDP.” Journal of Monetary Economics, 46: 79-95.

Nelson, C. R., and H. Kang. (1981). "Spurious periodicity in inappropriately detrended time series." Econometrica, 49: $741-751$.

Nelson, C. R., and C. Plosser. (1982). "Trends and random walks in macroeconomic time series." Journal of Monetary Economics, 10: 139-162.

Schenk-Hoppé, K. R. (2001a). "Random dynamical systems in economics.” Stochastics and Dynamics, 1: 63-83.

Schenk-Hoppé, K. R. (2001b). MatLAB scripts for business cycle simulation in the stochastic Solow model. Software available at the web page http://www.iew.unizh.ch/home/klaus/numerics/, Institute for Empirical Research in Economics, University of Zurich.

Schenk-Hoppé, K. R., and B. Schmalfuss. (2001). "Random fixed points in a stochastic Solow growth model." Journal of Mathematical Economics, 36: 19-30.

Solow, R. (1956). "A contribution to the theory of economic growth." Quarterly Journal of Economics, 70: 65-94.

Stadler, G. W. (1994). "Real business cycles." Journal of Economics Literature, 32: 1750-1783.

Stock, J. H., and M. W. Watson. (1999). "Business cycle fluctuations in US macroeconomic time series." In J. B. Taylor and M. Woodford (eds.), Handbook of Macroeconomics, vol. 1A. Amsterdam: Elsevier, pp. 3-64.

Swan, T. W. (1956). "Economic growth and capital accumulation." Economic Record, 32: 334-361. 